PROCEEDINGS OF THE

AMERICAN MATHEMATICAL SOCIETY

Volume 131, Number 11, Pages 3507-3509

S 0002-9939(03)06915-6

Article electronically published on February 14, 2003

\title{
A NOTE ON A THEOREM OF RAUBENHEIMER AND RODE
}

\author{
GERD HERZOG AND CHRISTOPH SCHMOEGER
}

(Communicated by Joseph A. Ball)

\begin{abstract}
We prove the converse of Raubenheimer and Rode's Banach algebra version of the Perron-Frobenius Theorem.
\end{abstract}

Let $(A,\|\cdot\|)$ be a complex Banach algebra with unit $e$ and $\|e\|=1$. A set $W \subseteq A$ is called an algebra wedge in the case that $W$ is closed, $W+W \subseteq W, \lambda W \subseteq W$ $(\lambda \geq 0), W \cdot W \subseteq W$, and $e \in W$. As usual, by setting $a \leq b: \Longleftrightarrow b-a \in W$ we obtain a reflexive and transitive relation on $A$, and call $A$ ordered by $W$. In the sequel let $\sigma_{A}(a)$ and $r(a)$ denote the spectrum and the spectral radius of $a \in A$, respectively.

In 2 Raubenheimer and Rode proved the following version of the PerronFrobenius Theorem.

Theorem 1. Let $A$ be ordered by an algebra wedge $W$ such that the spectral radius is increasing on $W$. Then $r(a) \in \sigma_{A}(a)$ for all $a \in W$.

The purpose of this note is to prove the converse of this result:

Theorem 2. Let $a \in A$ be such that $r(a) \in \sigma_{A}(a)$. Then there exists an algebra wedge $W$ such that the spectral radius is increasing on $W$, and $a \in W$.

Proof. For $S \subseteq A$ let $\Gamma(S)$ denote the centralizer of $S$, that is,

$$
\Gamma(S)=\{v \in A: v u=u v(u \in S)\},
$$

and set $B=\Gamma(\Gamma(\{a\}))$. Then $B$ is a closed commutative subalgebra of $A$, and $e, a \in B$. Moreover $\sigma_{A}(u)=\sigma_{B}(u)(u \in B)$ (see for example [3], Theorem 11.22). In particular $r(u)$ does mean the same in $A$ and $B$. Let $\Delta$ denote the set of all nontrivial multiplicative linear functionals on $B$.

Since $B$ is commutative and $r(a) \in \sigma_{B}(a)$ there is a $\psi \in \Delta$ such that $\psi(a)=r(a)$. We define

$$
W:=\{u \in B: r(u)=\psi(u)\} .
$$

Obviously $a \in W$, and $e \in W$ since $\psi(e)=1$. To see that $W$ is an algebra wedge first note that whenever $u, v \in B$ then, by Theorem 11.23 in [3],

$$
r(u+v) \leq r(u)+r(v), \quad r(u v) \leq r(u) r(v) .
$$

Moreover $r(u)=\max _{\varphi \in \Delta}|\varphi(u)|(u \in B)$.

For $u, v \in W$ we obtain

$$
r(u v) \leq r(u) r(v)=\psi(u) \psi(v)=\psi(u v) \leq r(u v),
$$

Received by the editors June 4, 2002 .

2000 Mathematics Subject Classification. Primary 45H05.

(C)2003 American Mathematical Society 
hence $u v \in W$, and analogously $u+v \in W$. Next, for each $u \in B$ and $\lambda \geq 0$ we have $r(\lambda u)=\lambda r(u)$, hence $\lambda W \subseteq W(\lambda \geq 0)$.

To prove that $W$ is closed, note that $(*)$ implies

$$
|r(u)-r(v)| \leq r(u-v) \leq\|u-v\| \quad(u, v \in B) .
$$

If $\left(u_{n}\right)$ is a convergent sequence in $W$ with limit $v$, say, then

$$
\begin{gathered}
\lim _{n \rightarrow \infty} \psi\left(u_{n}\right)=\psi(v), \\
\left|\psi\left(u_{n}\right)-r(v)\right|=\left|r\left(u_{n}\right)-r(v)\right| \leq\left\|u_{n}-v\right\| \rightarrow 0 \quad(n \rightarrow \infty),
\end{gathered}
$$

and therefore $r(v)=\psi(v)$. Altogether $W$ is an algebra wedge.

Now let $A$ be ordered by $W$. It remains to prove that $r$ is increasing on $W$. Let $0 \leq u_{1} \leq u_{2}$. Then

$$
r\left(u_{2}\right)-r\left(u_{1}\right)=\psi\left(u_{2}-u_{1}\right)=r\left(u_{2}-u_{1}\right) \geq 0 \Longrightarrow r\left(u_{1}\right) \leq r\left(u_{2}\right) .
$$

Examples. Consider $A=\mathbb{C}^{2 \times 2}$ and

$$
a:=\left(\begin{array}{cc}
0 & 1 \\
1 & 0
\end{array}\right), \quad r(a)=1 \in\{-1,1\}=\sigma_{A}(a) .
$$

In this case it is easy to check that

$$
B=\left\{u=\left(\begin{array}{ll}
\alpha & \beta \\
\beta & \alpha
\end{array}\right): \alpha, \beta \in \mathbb{C}\right\},
$$

and that $\psi(u)=\alpha+\beta$ is in $\Delta$ with $\psi(a)=1$. Since

$$
\sigma_{B}\left(\left(\begin{array}{cc}
\alpha & \beta \\
\beta & \alpha
\end{array}\right)\right)=\{\alpha+\beta, \alpha-\beta\}
$$

we get

$$
W=\left\{\left(\begin{array}{cc}
\alpha & \beta \\
\beta & \alpha
\end{array}\right):|\alpha-\beta| \leq \alpha+\beta\right\} .
$$

The same considerations for

$$
a:=\left(\begin{array}{cc}
0 & 1 \\
0 & 0
\end{array}\right), \quad r(a)=0 \in\{0\}=\sigma_{A}(a)
$$

lead to

$$
B=\left\{u=\left(\begin{array}{cc}
\alpha & \beta \\
0 & \alpha
\end{array}\right): \alpha, \beta \in \mathbb{C}\right\}, \quad \psi(u)=\alpha,
$$

and

$$
W=\left\{\left(\begin{array}{cc}
\alpha & \beta \\
0 & \alpha
\end{array}\right): \alpha \geq 0\right\} .
$$

In the first example $W$ is an algebra cone, that is, in addition $W \cap(-W)=\{0\}$. In the second example $W$ is not an algebra cone since $a \in W \cap(-W)$. This is not an accident, as the following results will show.

The radical of $A$, denoted by $\operatorname{rad}(A)$, is the intersection of all maximal ideals in $A$, and $A$ is called semisimple in case $\operatorname{rad}(A)=\{0\}$. It follows from [3], Theorem 11.9 , that if $A$ is commutative, then

$$
\operatorname{rad}(A)=\{u \in A: r(u)=0\} .
$$


Theorem 3. Let $a \in A, r(a) \in \sigma(a)$, and let $B, \psi$ and $W$ be as in the proof of Theorem 2. Then $\operatorname{rad}(B)=W \cap(-W)$. In particular $W$ is an algebra cone if and only if $B$ is semisimple.

Proof. 1. If $u \in \operatorname{rad}(B)$, then

$$
\sigma(u)=\{\varphi(u): \varphi \in \Delta\}=\{0\} .
$$

Thus $\psi(u)=0=r(u)$, hence $u \in W$. We have $-u \in \operatorname{rad}(B)$, $\operatorname{since} \operatorname{rad}(B)$ is an ideal in $B$. As above we conclude $-u \in W$. Therefore $u \in W \cap(-W)$.

2. Let $u \in W \cap(-W)$. Then

$$
\psi(u)=r(u)=r(-u)=\psi(-u)=-\psi(u) .
$$

Hence $\psi(u)=r(u)=0$, and therefore $u \in \operatorname{rad}(B)$.

As usual, an algebra cone is called normal if there exists a constant $\alpha \geq 1$ such that $0 \leq u \leq v$ implies $\|u\| \leq \alpha\|v\|$.

If $A$ is a $C^{*}$-algebra, then $a \in A$ is called normal if $a a^{*}=a^{*} a$. In this case we have $r(a)=\|a\|$ (see for example [1], Theorem 58.3). As a consequence of Theorem 3 we get the following result.

Theorem 4. Suppose $A$ is a $C^{*}$-algebra, $a \in A$ is normal, and $r(a) \in \sigma(a)$. Let $W$ be the algebra wedge from the proof of Theorem 2. Then:

(1) $W$ is a normal algebra cone with constant $\alpha=1$;

(2) each $u \in W$ is normal.

Proof. Let $B, \psi$ and $W$ be as in the proof of Theorem 2. By the Fuglede-Putnam Theorem (see for example [1], Exercise 65.1) $\Gamma(\{a\})$ is a $*$-subalgebra of $A$, thus $B=\Gamma\left(\Gamma\left(\left\{a, a^{*}\right\}\right)\right)$, and therefore each $u \in B$ is normal. This shows that (2) is valid, and that $\operatorname{rad}(B)=\{0\}$. Hence $B$ is semisimple, and $W$ is an algebra cone according to Theorem 3. Finally let $0 \leq u \leq v$. Since $r$ is increasing on $W$ we have

$$
\|u\|=r(u) \leq r(v)=\|v\| \text {. }
$$

\section{REFERENCES}

1. Berberian, S.K.: Lectures in functional analysis and operator theory. Graduate Texts in Mathematics, Springer-Verlag, New York, 1974. MR 54:5775

2. Raubenheimer, H., Rode, S.: Cones in Banach algebras. Indag. Mathem. 7 (1996), 489-502. MR 99i: 46035

3. Rudin, W.: Functional analysis. Second edition. International Series in Pure and Applied Mathematics. McGraw-Hill, Inc., New York, 1991. MR 92k:46001

Mathematisches Institut I, Universität Karlsruhe, D-76128 Karlsruhe, Germany

E-mail address: Gerd.Herzog@math.uni-karlsruhe.de

Mathematisches Institut I, Universität Karlsruhe, D-76128 Karlsruhe, Germany

E-mail address: Christoph.Schmoeger@math.uni-karlsruhe.de 